\title{
DEVELOPMENT OF A LOW-COST, HAND-HELD MULTI-SENSOR SYSTEM FOR THE MONITORING OF SMALL WATER BODIES
}

\author{
R. Blaskow ${ }^{1 *}$, E. Schwalbe ${ }^{1}$ \\ ${ }^{1}$ Institute of Photogrammetry and Remote Sensing, Technische Universität Dresden, Germany \\ (robert.blaskow, ellen.schwalbe)@tu-dresden.de
}

Commission I, WG I/7

KEY WORDS: mobile mapping, mobile laser scanning, mono visual odometry

\begin{abstract}
:
For small-scale monitoring of small water bodies, conventional methods such as GNSS or total station measurements are used. The data acquisition is usually carried out in profile form supplemented with extra measurements of break edges, slope edges or bank courses. However, these methods can be used efficiently only on small sections and with low temporal resolution. At the same time, as the length of rivers or creeks to be monitored increases, the cost-effectiveness of the above methods decreases. Further limitations such as very small sections that are difficult to access and also sections that are sometimes heavily overgrown also prevent the use of large measuring platforms. By contrast, with use of a hand-held compact multi-sensor platform it is possible to survey several hundred kilometres of the smallest rivers and creeks. This publication demonstrates the use of such a platform to record microwatersheds. For this purpose, the Creek4D project, the measurement principle and the sensor technology used are shown. In addition, first measurement data and the calibration strategy are shown.
\end{abstract}

\section{INTRODUCTION}

The Creek4D project is developing a new method for surveying small urban water bodies, some of which are difficult to access. The goal is to create a data basis that can be used for planning measures for achieving a good ecological status of these water bodies, for management purposes and flood protection. This requires a small-scale and high-resolution description of the small rivers and creeks morphological conditions. The collection of such data is very time-consuming and costintensive, when using conventional surveying methods. Figures from the Dresden Environmental Agency, which is involved in this project, show that there are a total of $500 \mathrm{~km}$ of small rivers and creeks to be monitored in the Dresden urban area alone. A study has shown that the survey and structural assessment of these water bodies is possible for only $5-10 \mathrm{~km}$ per year using conventional methods. The most time-consuming factor here is the tachymetric measurement of the water bodies.

\subsection{State of the Art}

Recent projects show that multi-sensor mobile platforms are a very good tool for recording geometric data of built and natural structures. Thus, depending on the object characteristics, unmanned aerial (Mader et al., 2015) or water vehicles (Sardemann et al., 2018) can be used. In our case the use of these platforms is not practicable because the creeks to be measured are partly very small and heavily overgrown. The overgrowth prevents a clear view from the air and the partly very low water level does not allow for the use of a boat. Therefore, a hand-held mobile platform is being developed for this purpose.

\footnotetext{
* Corresponding author
}

\section{METHOD}

\subsection{Measurement procedure}

The basic concept of data acquisition is the measurement of the creek course by continuously moving a sensor system along an imaginary creek axis. If the water level is low, the sensor system should be moved directly along the creeks middle axis, if the creek bed is inaccessible, it should be moved slightly offset from the axis in the bank area. The creek to be measured is divided into individual sectors, as shown in Figure 1. These are measured twice on an outward and return path.

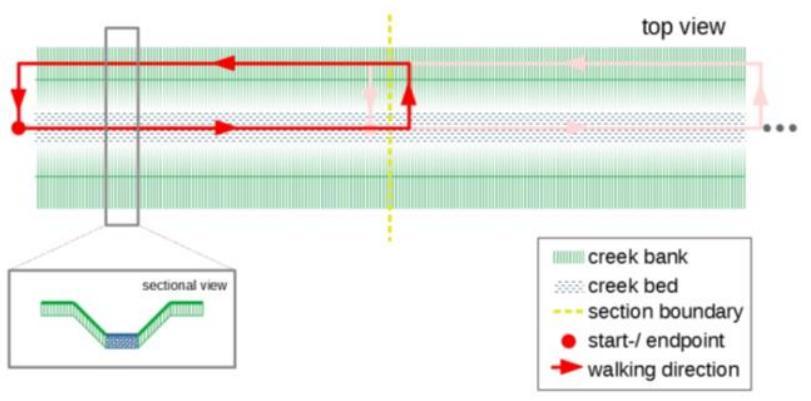

Figure 1. Principle of the measurement procedure

This measuring procedure is based on the usual recording principle of SLAM-based measuring systems. For a high accuracy of the trajectory to be determined, a so-called loop closure is necessary. This means that the start and end points of the measurement must be identical (Figure 1). In addition to the higher geometric stability of the trajectory, this method also has the advantage that possible shadowing of the laser scanner signal is minimized by traversing the sector in two different 
height levels, and the point density of the resulting point cloud is increased. Especially vegetation close to the ground can be better penetrated when scanning twice from slightly different perspectives. To avoid gaps in the resulting data, a slight overlapping of the single measurements at the sector boundaries should also be performed (Figure 1 top view). In the case of very dense vegetation along the creek course, more scan paths can be measured in addition to the outward and return path to increase the point density and coverage. Leaving the creek axis due to obstacles is also possible with this measuring method.

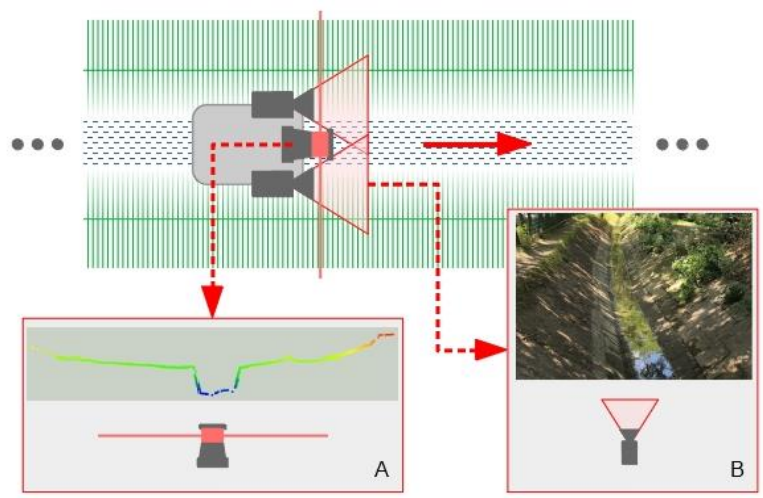

Figure 2. (on top) Simplified representation of the sensor system. (below) Sample data of the scanner (A) and camera (B).

In addition to a 2D laser scanner (Figure 2A), an RGB camera (Figure 2B) is installed on the platform. This is used on the one hand for supporting the orientation of the mobile platform and on the other hand for coloring the laser scanner point cloud.

\subsection{Mobile platform}

The first version of a mobile test carrier is based on a plywood construction, which is reinforced with individual pine wood elements. The size of the basic carrier corresponds to DIN A5 format. Table 1 provides an overview of the components currently installed on it.

\begin{tabular}{|l|c|}
\hline Model & Component \\
\hline Laser-scanner & Hokuyo UTM-30LX-EW \\
Camera & Raspberry HQ camera (f=6mm) \\
IMU & MPU-9250 \\
GPS & Ublox-NEO-6M-GPS \\
Data processor & Raspberry Pi 3 Model B \\
Power supply & Powerbank 5V + Powerbank 12V \\
\hline
\end{tabular}

Table 1. System components

The laser scanner projecting to the front increases the length of the overall construction to $25.5 \mathrm{~cm}$. The double bottom houses the power supply in the form of two power banks. On the base plate a Raspberry Pi is located which controls the measuring components. Attached to the reinforced central construction are the inertial measurement unit (IMU), the laser scanner and the camera.

As already described, the laser scanner is tilted $90^{\circ}$ to the front (Figure 3A). Approximately $90^{\circ}$ of the viewing area of the currently installed Hokuyo laser scanner is occluded by the housing. No measurement data can be generated in this area (Figure 3B).

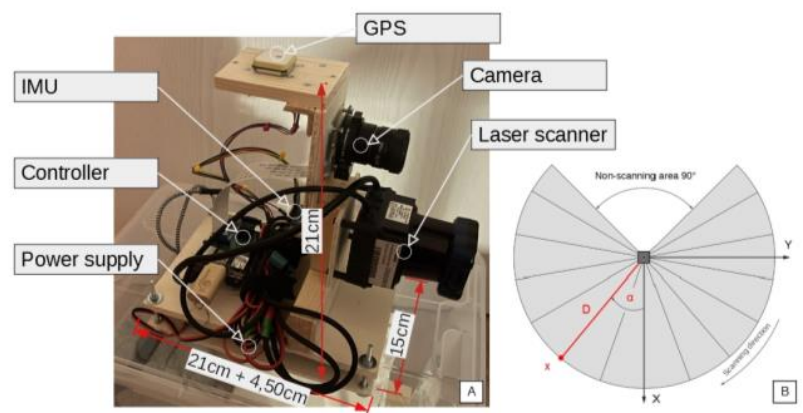

Figure 3. (A) Developed test platform and (B) schematic representation of the scan area.

Due to the higher priority of the ground recording, the scanner was rotated so that the internal primary coordinate axis points downwards and the occluded area points upwards. A Raspberry HQ camera is installed to support the platform orientation. The connection of this camera to the camera serial interface (CSI-2) simplifies the time synchronization with the other sensors. The IMU and the GPS module is connected via the i2c and serial interface of the Raspberry respectively.

\subsection{Data processing}

In contrast to some commercially available systems, the platform designed here is only used for data acquisition. The omission of on-board data processing has both advantages and disadvantages, but the advantages outweigh the disadvantages for the requirements of this project. The main disadvantage is that during and after the measurement in the field, the plausibility and quality of the recorded data cannot be controlled immediately. However, this can be remedied by detailed instructions for the measurement procedure. The main advantage, on the other hand, is that a much cheaper, more robust and power-saving control unit can be installed for pure data recording. This will make the system more compact, lighter and easier to operate. The use of standard components will also make the system much easier to repair. The aim is to create a robust, easy-to-operate and maintain measuring system.

\subsection{Platform orientation methods}

Several methods will be investigated for the orientation of the mobile platform. With the single camera available on the test platform, the suitability of image sequences of these natural environments for navigation will first be tested using mono visual odometry (e.g. Forster et al., 2014). In a second step and if the image data is suitable, a second camera will be added to the platform to form a stereo camera system to tackle the scale factor issue of the monocular odometry estimation. In the next step, the suitability of IMU and GNSS sensors for the orientation of the platform will be investigated separately and in combination with the image data. When all sensors have been checked for suitability, the system calibration between GNSS/IMU, camera and laser scanner is carried out. The data readout required for this and the temporal synchronization of the measurement data is already implemented.

\subsection{Calibration procedure}

As described above, the orientation and calibration of the mobile platform is performed in several steps. As a first step, the relative orientation (lever-arm and boresight) between camera and 2D laser scanner will be determined. 
For this purpose, a modified version of the integrated bundle adjustment presented in (Mader et al., 2014) is used. To generate suitable input data, the reference field used in (Mader et al., 2014) needed to be modified. As described in section 2.2 the field of view of the camera and that of the laser scanner do not overlap. Thus, the reference point field must consist of camera and laser scanner related components that can be measured in one coordinate-system.

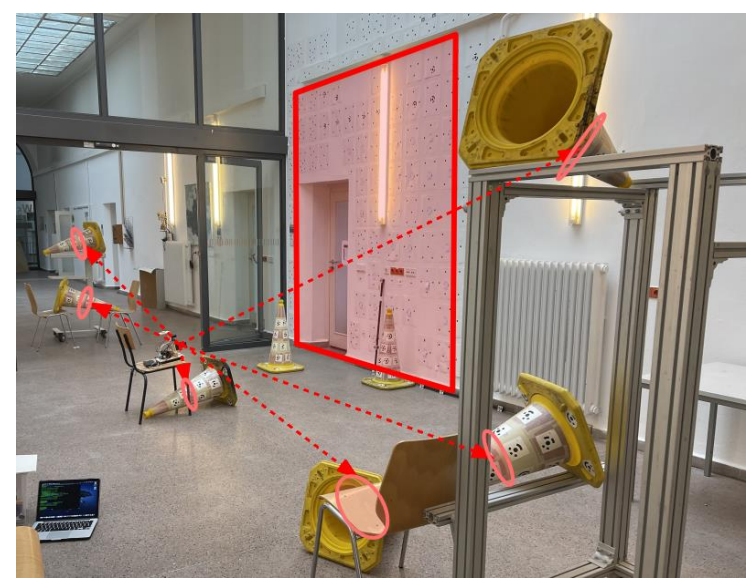

Figure 4. Reference field consisting of (non)-coded targets on the wall (CAM) and with coded targets signalized cones (LS)

A wall signalized with measurement marks on a large scale for the determination of the camera positions (Figure 4 red square) and a spatial measurement field for the 2D laser scanner consisting of cones signalized with coded measurement marks (Figure 4 light red ellipses) were combined to one reference field.

\section{EXPERIMENTS AND ONGOING WORK}

Since the GNSS data is not yet usable, the platform positions are determined from camera images using Structure from motion reconstruction. Besides that, additional investigations were undertaken to eliminate the scale problem. The goal was to produce a scaled platform trajectory from the image data using Structure from motion with as little reference information as possible. Together with the determined lever-arm and boresight, this camera-based trajectory should be used for the orientation of the laser scanner.

A monument with a size of $6.5 \times 1 \mathrm{~m}$ and a height of $3 \mathrm{~m}$ served as measurement object and was recorded with the mobile platform according to the procedure described in section 2.1. To obtain reference data for the test object it was additionally scanned with a terrestrial laser scanner (TLS) The extent of the test object is smaller than the planned length of the river sections to be recorded later and the reconstructability of the geometric object is better than it would be for a natural measurement setting. Since the 2D laser scan profiles are computationally attached to the camera positions, the initial focus is on the highest possible quality of the reconstructed camera position and small distances between them. The scaling of the calculated trajectory was realised by using two scale bars, which were only visible on the outward path of the measurement. To determine the camera positions, a 3D reconstruction of the recorded scene was conducted using Structure from Motion (SfM). The quality of the reconstruction was derived from the point to point distances between the reference TLS point cloud and the SfM point cloud as shown in Figure 5. To enable comparability between the point clouds, present in different local systems, the SfM point cloud was previously registered to the reference TLS point cloud without estimating the scale. In this way, a conclusion can be drawn about the quality of the reconstructed SfM points from the fitting accuracy between the two point clouds.

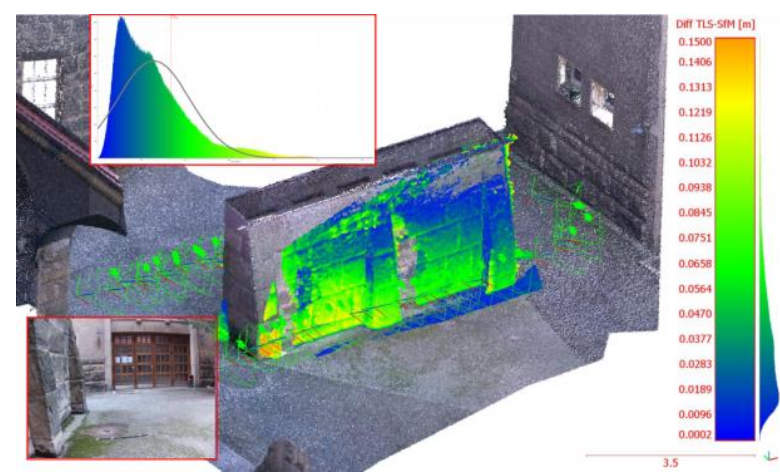

Figure 5. Point to point difference between TLS Reference point cloud (RGB) and SfM point cloud

From Figure 5 it can be seen that a reconstruction was possible from the recorded image sequence. Here it can be seen that $96 \%$ of the point distances of the wall points, lying within the trajectory, are less than $0.10 \mathrm{~m}$ and $74 \%$ of the distances are less than $0.05 \mathrm{~m}$. The standard deviation of the point distances is $0.0248 \mathrm{~m}$ in the considered area. A visual interpretation of the colour gradient of the wall points shows a slight systematic effect, which results from the tilt of the SfM point cloud in walking direction. A similar effect has occurred on the back side of the wall, which is not shown here.

With respect to the relatively unfavourable acquisition configuration, the low cost camera used and the reference introduced only by two scales in the outward path of the measurement, the result can be rated as acceptable. In a further step the camera locations can now be used as initial positions for the laser scanner profiles. Precondition for this is the successful determination of the relative orientation between camera and laser scanner.

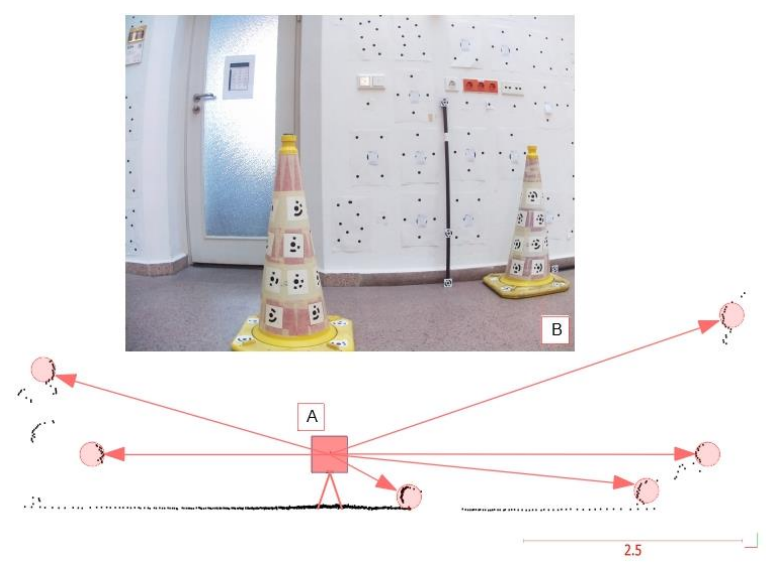

Figure 6. Sample from a first measurement campaign Raspberry Pi HQ Cam (B) and Hokuyo LS Scan (A)

To perform a first feasibility study of the calibration procedure presented in section 2.5 , measurement data has already been 
generated in a measurement campaign. Therefore, the mobile measurement platform was set up at seven different locations along the alignment between the cones in the described reference field. Figure 6 shows the camera image and the 2D scan profiles of the laser scanner (LS) from one measurement position. Due to the small number of cones, it was difficult to vary the mobile platform positions or even to rotate the mobile platform against the cone alignment. Likewise, the spatial coverage of the 2D scan profile by the cones (Figure 6A) is still insufficient. Improvements are currently being worked on at this point.

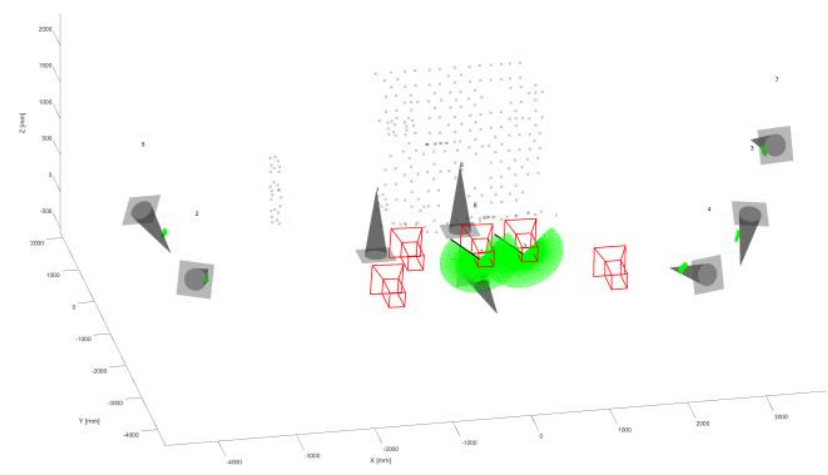

Figure 7. Visualization of the network geometry: Consisting of the cones for LS measurement, four camera poses, two LS poses (green fans) and the wall targets (dots)

From these data, two viewpoints of the mobile platform were selected for the feasibility study. As already mentioned in section 2.5, an adapted version of the bundle block adjustment of (Mader et al., 2014) was used to calculate the positions of the laser scanner projection centre and the values for lever-arm and boresight of the laser scanner to the camera. Figure 7 shows the result graphically. In addition to the already explained reference field (cone and wall reference points), four camera and two laser scanner poses (green fans) are shown.

\begin{tabular}{|c|c|c|c|c|}
\hline & \multicolumn{2}{|c|}{ LS Pose 1 } & \multicolumn{2}{c|}{ LS Pose 2 } \\
\hline & Value & Accuracy & Value & Accuracy \\
\hline $\mathrm{X}[\mathrm{mm}]$ & -158.276 & 2.855 & 398.294 & 2.855 \\
$\mathrm{Y}[\mathrm{mm}]$ & -2105.206 & 6.268 & -2084.091 & 6.474 \\
$\mathrm{Z}[\mathrm{mm}]$ & 555.323 & 3.532 & 570.371 & 3.646 \\
$\mathrm{o}[\mathrm{deg}]$ & 96.864 & 0.581 & 101.692 & 0.803 \\
$\mathrm{p}[\mathrm{deg}]$ & -0.448 & 4.710 & -0.637 & 3.982 \\
$\mathrm{k}[\mathrm{deg}]$ & -87.060 & 0.574 & -86.986 & 0.784 \\
\hline
\end{tabular}

Table 2. Results of the first feasibility study with two LS poses

In Table 2, from all the calculation results of the bundle block adjustment, the exterior orientation of the two laser scanner poses, which is important here, and their accuracy are shown. It can be seen here that both, the position and the rotation components have their largest standard deviation values in the Y-direction. One factor for these differences between XZ-and Y-direction may be the insufficient recording configuration. For example, only two poses of the mobile platform were used for the calculation, which do not offer a large variation in the $\mathrm{XZ}$ direction. The reference field, which is still to be optimized, did not allow any variation of the positions in the $\mathrm{Y}$ direction. Furthermore, the still insufficient coverage of the entire scanning area of the laser scanner with cones also affects the accuracy of the position determination.

\begin{tabular}{|c|c|c|c|}
\hline & & Value & Accuracy \\
\hline \multirow{3}{*}{ 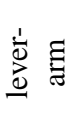 } & $\mathrm{X}[\mathrm{mm}]$ & -19.278 & 8.420 \\
\hline & $\mathrm{Y}[\mathrm{mm}]$ & -89.544 & 5.752 \\
\hline & $\mathrm{Z}[\mathrm{mm}]$ & -31.321 & 13.458 \\
\hline \multirow{3}{*}{ 总 } & o [deg] & -171.969 & 2.083 \\
\hline & $\mathrm{p}$ [deg] & 0.154 & 2.174 \\
\hline & $\mathrm{k}$ [deg] & 80.482 & 1.012 \\
\hline
\end{tabular}

Table 3. Results of the first feasibility study for boresight and lever-arm between platform camera and laser scanner

The limitations mentioned above are also reflected in the results of the lever-arm and boresight alignment between the platform camera and laser scanner. The inaccuracies of the camera and laser scanner positioning led to high deviations of $13.5 \mathrm{~mm}$, especially in the $\mathrm{Z}$ component.

\section{SUMMARY AND OUTLOOK}

The purpose of this publication is to present the Creek4D project, the first steps of the development of a mobile multisensor platform and the workflow for the efficient measurement of small water bodies. First measurements could show the potential of the low cost components. It is possible to reconstruct the trajectory of the mobile platform with images from a Raspberry Pi HQ camera and a minimum of reference information, in order to use these as poses for the 2D laser scanner profiles. Likewise, a first successful calculation of the relative orientation between camera and laser scanner could be performed. Future work will now be to improve these results. For this purpose, a modification of the reference field will be carried out.

The next steps will be the integration of the IMU measurement values into the trajectory determination procedure and the extension of the camera system.

\section{ACKNOWLEDGEMENTS}

In cooperation with the Umweltamt Dresden and funded by the DBU (Deutsche Bundesstiftung Umwelt).

\section{REFERENCES}

Forster, C., Pizzoli, M., \& Scaramuzza, D., 2014. SVO: Fast semi-direct monocular visual odometry. In 2014 IEEE international conference on robotics and automation (ICRA) (pp. 15-22). IEEE.

Mader, D., Westfeld, P., \& Maas, H. G., 2014. An Integrated Flexible Self-calibration Approach for 2D Laser Scanning Range Finders Applied to the Hokuyo UTM-30LX-EW. The International Archives of Photogrammetry, Remote Sensing and Spatial Information Sciences, 40(5), 385.

Mader, D., Blaskow, R., Westfeld, P., \& Maas, H. G., 2015. UAV-based acquisition of $3 \mathrm{~d}$ point cloud-A comparison of a low-cost laser scanner and SFM-tools. The International Archives of Photogrammetry, Remote Sensing and Spatial Information Sciences, 40(3), 335.

Sardemann, H., Eltner, A., \& Maas, H. G., 2018. Acquisition of geometrical data of small rivers with an unmanned water vehicle. International Archives of the Photogrammetry, Remote Sensing and Spatial Information Sciences, 42(2), 1023-1027. 